\title{
REASONING ABOUT SPACE: THE HOLE STORY
}

\author{
CONTENTS
}

\author{
1. Introduction, p. 4 \\ 2. The Basic Theory, p. 5 \\ 3. Examples and Developments: \\ the Geometry of Containment, p. 27
}

Received May 10, 1995. Revised April 15, 1996 


\section{Introduction}

Much of our naive reasoning a bout space involves reasoning about holes and holed objects. We put things in holes, through holes, around them; we jump out of a hole or fall into one; we compare holes, measure them, enlarge them, fill them up.

What exactly holes are, or even whether such dubious entities do indeed exist, these are of course questions one eventually needs to address in order to make good sense of such forms of reasoning. For instance, treating holes as (parts of) material objects, say, by identifying them with hole-linings [21], would call for an account of the altered meaning of certain predicates or prepositions. (What would 'inside' and 'outside' mean? What would it mean to 'enlarge' a hole?) On the other hand, a non-realist attitude would require some radical eliminative strategy, some systematic way of paraphrasing every hole-committing sentence by means of a sentence that does not refer to or quantify over holes [20] (the cheese is holed, but there is no hole in it). This might well be a favorable strategy, provided the language contain all the necessary shape-predicates: after all, holes are a paradigm example of nothings.

The general philosophical hypothesis underlying the present work is that one should resist these ways out in favor of a realist, common-sense attitude. Holes are enigmatic, and it may be utterly difficult to specify adequate identity and individuation criteria for them. Yet, if there is an ontology inherent in our everyday reasoning about the world, then this ontology comprises holes (and cognate entities such as cracks, cuts, or fissures) along with stones and chunks of cheese. This view stems from joint work with Roberto Casati and has been largely presented in [3], where a basic formalism is also introduced to spell out the major tenets and consequences of a realist theory. The purpose of this paper is to elaborate on that formalism and to illustrate how it can be exploited to provide a framework for more general patterns of qualitative spatial reasoning. In particular, after a general outline, I shall focus on some issues pertaining to the modelling, the representation, and the taxonomy of spatial inclusion. This is a topic that has received much attention in recent work in spatial reasoning, also in connection to a variety of applications, from naive physics to natural language semantics. My hope is to show that explicit commitment to holes as bona fide individuals introduces a novel and revealing (albeit certainly far from complete) perspective also with respect to such issues. 


\section{The Basic Theory}

I shall begin with the general picture: countenancing holes calls for some explicit theory (i.e., set of principles) about these entities - their identity conditions, their part-whole relations, their patterns of interaction with the environment. The picture will be necessarily schematic and in large strokes, and I shall have to refer to previous work for the underlying motivations. I shall, however, take the opportunity to introduce some novelties and refinements into the account which will result into a simplified and somewhat more refined theory - particularly for the purpose of qualitative spatial reasoning - than the one originally outlined in [3].

Various independent domains come to interact in the development of this general picture. Focusing on the extensional core, four main domains may be distinguished:

- ontology (holes are parasitic entities);

- mereology (holes may bear part-whole relations to one another);

- topology (holes are one-piece things located at the surfaces of their hosts); - morphology (holes are fillable, and can be penetrated by other objects).

I shall deal with these domains in turn, working within the framework of a first-order language with descriptions. The underlying logical machinery will be deliberately left vague. (In effect, why should holes make a difference?) A preferred option is a free quantification theory with some means for dealing with improper descriptions, but a more classical framework might provide a viable basis as well. As for the notation, I shall use ' $\neg$ ', ' $\wedge$ ', ' $\vee$ ', ' $\rightarrow$ ', and ' $\leftrightarrow$ ' as connectives respectively for negation, conjunction, disjunction, material implication, and material equivalence (in decreasing order of strength); ' $\forall$ ' and ' $\exists$ ' for the universal and existential quantifiers; and ' $\imath$ ' for the definite descriptor. As a rule, the outermost quantifiers in universally quantified formulas will be omitted to simplify readability.

2.1. Ontology. Holes are sui generis. They are spatiotemporally located, like stones and chunks of cheese, but they are not made of anything. They are not just regions of space either; for holes can move (as happens anytime you move a piece of Swiss cheese), whereas spatial regions cannot. In fact holes are always in or through something else, and this makes them ontologically parasitic entities: they cannot exist without (or be removed from) a material host. You can't have the hole without the doughnut, so to say.

This form of ontological dependence likens holes to other underpriviledged entities, such as surfaces or other boundary elements (see e.g. [6]). It should be distinguished from the purely conceptual or de dicto dependence 
exemplified by sentences such as "every sister has a sibling": there cannot exist sisters without siblings, but it is not true of any sister that she could not have existed as an only child. By contrast, a hole cannot exist except as a hole in something, so it suffers from a form of truly de re dependence. (This also explains the difference between the dependence of the hole on the doughnut vs. the dependence of the latter on the former. Surely you cannot have a holeless doughnut. But that is a form of conceptual dependence, nothing more. If you cut your doughnut into pieces, the hole goes, the doughnut stays - though its shape is now different.) This form of de re dependence in turn should be distinguished from stronger forms, such as the dependence of a grimace on a face. That grimace can only exist as an expression on that face. But one can in principle change the host's matter, its shape, or even the entire host without affecting the hole in it. In other words, holes are not rigidly dependent on their hosts (in the terminology of [34]). Something is a hole if and only if it is a hole in something.

It is of course impossible to do justice to these distinctions without resorting in some way to modal notions. However, for our purposes the opposition between rigid and generic dependence is not essential, and we can initially content ourselves with emphasizing the de dicto/de re opposition. This could be done directly, by assuming a primitive relation of dependence and then asserting that all holes (entities of a kind) bear that relation to some material objects. (The relation will have to be governed by suitable axioms, e.g. along the lines of [12].) Alternatively, we can simply express the desired feature by taking as a starting point a relational primitive, say ' $\mathrm{H}(x, y)$ ', to be read as " $x$ is a hole in (or through) $y$ ". This is the strategy followed in [3], and I shall adhere to it in the present context. We may lose something in expressive power, but it goes straight to the point: there are no holes simpliciter - holehood is a relational property. An interesting generalization would be to rely on a three-place relation, ' $\mathrm{H}(x, y, z)$ ', meaning " $x$ is a hole in (or through) $y$ relative to $z$ ". This would do justice to the intuition that the notion of a hole is somewhat context-dependent. (A roof-window is a hole in the roof in some respect, but not in others. It lets the light in, not the rain.) However I shall not pursue this line of generalization, for reasons of simplicity and also because the relevant distinctions can eventually be dealt with by taking into account the way a hole can be filled (Section 2.4 below). A see-through filler is no light deterrent, but it stops the rain; a cloth filler will make a nice curtain, but it'll rain in.

Let then ' $\mathrm{H}(x, y)$ ' stand for " $x$ is a hole in (or through) $y$ ". We take this relation to be governed by the following basic axiom, which defines the main constraint on the hole-host relationship: 
AH1

$$
\mathrm{H}(x, y) \rightarrow-\mathrm{H}(y, z) .
$$

That is, the host of a hole is not itself a hole. It follows immediately that $\mathrm{H}$ is irreflexive and asymmetric; no hole is a hole in itself, and there is no room for holey loops:

TH1

$$
\neg \mathrm{H}(x, x)
$$

TH2

$$
\mathrm{H}(x, y) \rightarrow-\mathrm{H}(y, x) .
$$

Moreover, AH1 suffices to certify some basic facts about holes. For instance, holes are holeless, i.e., cannot host other holes, and cannot exist unless some other sort of entity also exists:

TH3

$$
\begin{array}{ll}
\text { TH3 } & \mathrm{H}(x, y) \rightarrow-\mathrm{H}(z, x) \\
\text { TH4 } & \exists_{x} \exists_{y} \mathrm{H}(x, y) \rightarrow \exists_{x} \neg \exists_{y} \mathrm{H}(x, y) .
\end{array}
$$

This is not to deny that a hole may be part of another hole (contrary to [2]). Nor does TH3 rule out the possibility that a hole can be located inside another hole. Imagine placing a holed piece of Gruyère inside a hole in a bigger chunk of Emmenthaler. Or think of the topologists" "hole through the hole in the hole": a hole connects one face of a cube to an opposite face after bifurcating into two separate channels for part of the way, and a second hole passes between the two channels to connect two other faces. Such situations are perfectly consistent with the view that a hole cannot be $a$ hole in another hole. Every hole needs a material host and cannot, therefore, be the host of other holes. But holes are immaterial and can, therefore, be interpenetrated by other entities - including other holes. Something can be spatially enclosed in a hole without being a part thereof. The rest of the theory is concerned precisely with these situations - with the multiple ways a hole can bear spatial relations with other things, be they immaterial bodies, material objects, or simply regions of space.

2.2. Mereology. The principles governing the relevant part-whole relationships can be formulated within the framework of formal mereology, eventually supplemented with specific axioms on $\mathrm{H}$. The parts of a hole are not in the hole in the same sense in which the hole is in its host; and the relationship of a hole to its host is not a relation of part to whole. Putting a coin in the hole of a doughnut does not make it become part of the doughnut. Nor does the coin occupy part of the doughnut - say, a "negative" part, as some have suggested [19]. Holes have nothing in common with their hosts, however negatively you look at them, and the holehood relation must be supplemented by an independent parthood relation. (This is not uncontroversial, and I refer to [5] for further elaborations.) 
The exact format of the background part-whole apparatus is arguably not quite binding. That is, it should be independently motivated, and I should like to think that hole-related reasoning is compatible with a variety of mereological theories. For the sake of definiteness, however, I shall here rely on a version of classical extensional mereology based on the primitive relation " $x$ is a (possibly improper) part of $y$ ", written ' $\mathrm{P}(x, y)$ '. This has the following axioms

$$
\begin{array}{ll}
\text { AP1 } & \mathrm{P}(x, y) \leftrightarrow \forall_{z}(\mathrm{O}(z, x) \rightarrow \mathrm{O}(z, y)) \\
\text { AP2 } & \exists_{x} \varphi \rightarrow \exists_{z} \forall_{y}\left(\mathrm{O}(y, z) \leftrightarrow \exists_{x}(\varphi \wedge \mathrm{O}(x, y))\right),
\end{array}
$$

where ' $\mathrm{O}$ ' (overlap) is defined as usual as sharing of a common part:

$\mathrm{DP} 1 \quad \mathrm{O}(x, y)=_{\mathrm{df}} \exists_{z}(\mathrm{P}(z, x) \wedge \mathrm{P}(z, y))$.

Thus, by AP1 parthood amounts to inclusion of overlappers: this ensures that $\mathrm{P}$ be an extensional partial ordering (reflexive, antisymmetric, and transitive) whereas $\mathrm{O}$ is reflexive and symmetric, but not transitive. By AP2, every satisfied condition $\varphi$ picks out a unique entity consisting of all $\varphi$ ers, viz. their "fusion". This is perfectly standard and yields a classical extensional mereology in the sense of [34], corresponding to a Boolean algebra with the bottom element removed. As I said, other accounts are in principle compatible with the present purposes, but I prefer to rely on the classical account for definiteness and for reasons of simplicity. Of course this is not uncontroversial. In particular, AP2 is sometimes questioned on the grounds that it has counter-intuitive instances when $\varphi$ is true of scattered entities (each and every table, or my nose and your favorite restaurant). From a purely mereological prospective, however, I think this is not an issue. One may feel uncomfortable with treating unheard-of Goodmanian mixtures as individuals. But which individuals are more "natural" than others - which sums count as genuine wholes - is not a mereological question. In fact it is a question that cannot even be formulated in mereological terms, and requires reference precisely to the sort of topological notions considered below. (See $[42,43]$ for developments of this argument.)

Other derived notions that will be used in the following include proper parthood (PP), identity $(=)$, and the operations of general sum $(\sigma)$ and product $(\pi)$ justified by AP2, along with the usual quasi-boolean functors for binary sum $(+)$, product $(\times)$, difference $(-)$, and complement $(\sim)$ :

$$
\begin{array}{ll}
\mathrm{DP} 2 & \mathrm{PP}(x, y)=_{\mathrm{df}} \mathrm{P}(x, y) \wedge \neg \mathrm{P}(y, x) \\
\mathrm{DP} 3 & x=y={ }_{\mathrm{df}} \mathrm{P}(x, y) \wedge \mathrm{P}(y, x)
\end{array}
$$




$\begin{array}{llll}\text { DP4 } & \sigma x \varphi & =_{\mathrm{df}} & \imath z \forall_{y}\left(\mathrm{O}(y, z) \leftrightarrow \exists_{x}(\varphi \wedge \mathrm{O}(x, y))\right) \\ \text { DP5 } & \pi x \varphi & =_{\mathrm{df}} & \sigma z \forall_{x}(\varphi \rightarrow \mathrm{P}(z, x)) \\ \text { DP6 } & x+y & =_{\mathrm{df}} & \sigma z(\mathrm{P}(z, x) \vee \mathrm{P}(z, y)) \\ \text { DP7 } & x \times y & =_{\mathrm{df}} & \sigma z(\mathrm{P}(z, x) \wedge \mathrm{P}(z, y)) \\ \text { DP8 } & x-y & =_{\mathrm{df}} & \sigma z(\mathrm{P}(z, x) \wedge \neg \mathrm{O}(z, y)) \\ \text { DP9 } & \sim x & =_{\mathrm{df}} & \sigma z(\neg \mathrm{O}(z, x)) .\end{array}$

(The functors introduced by DP4-DP9 will of course be partially defined - i.e., they may correspond to improper descriptions unless we go with the fiction of a null individual that is part of everything, as in [23]. This introduces some complexities into the underlying logic: we may adopt a Russellian theory of descriptions (as in [22]), or we may opt for some free logic (compare [35]). Again, since these questions do not depend specifically on the application at issue, I shall leave them open.) We can also add some mixed notions, such as being a (proper) hole-part:

$\mathrm{DP} 10 \quad \mathrm{HP}(x, y)=_{\mathrm{df}} \mathrm{P}(x, y) \wedge \exists_{z} \mathrm{H}(x, z)$

$\operatorname{DP} 11 \quad \operatorname{PHP}(x, y)=_{\mathrm{df}} \operatorname{PP}(x, y) \wedge \exists_{z} \mathrm{H}(x, z)$.

Thus, a (proper) hole-part is a (proper) part which qualifies as a hole though not a hole in the same entity of which it is a part. (This is not ruled out by the definition, but will soon become apparent.)

On these grounds, the specific axioms on the interplay between $\mathrm{P}$ and $\mathrm{H}$ are now as follows. First of all, we have a sort of extensionality axiom, likening holehood to other ontologically fundamental properties. I have left it open whether a hole can have more than one host. In fact it can, given our general mereological set-up: a hole in the pocket is a hole in the jacket, if the pocket is part of the jacket. More importantly, however, we want to ensure that two distinct holes cannot share exactly the same hosts (at a given time) - that is, if two holes are distinct, one must always be able to cut any common host (if any) into two disjoint parts, one of which will host exactly one hole (not the other). More generally, the intended relation between holes and hosts is defined by the following axiom:

AP3

$$
\exists_{y} \mathrm{H}(x, y) \wedge \forall_{y}(\mathrm{H}(x, y) \rightarrow \mathrm{H}(z, y)) \rightarrow \mathrm{P}(z, x) .
$$

From which the advertised form of extensionality follows immediately via DP3:

TP1 $\quad \exists_{y} \mathrm{H}(x, y) \wedge \forall_{y}(\mathrm{H}(x, y) \leftrightarrow \mathrm{H}(z, y)) \rightarrow x=z$. 
As most extensionality principles, AP3 and TP1 may of course fail if we take parthood and identity as relations across time. You can destroy some of the hosts (e.g., by destroying some parts thereof) without affecting the identity of the hole. However, relative to a fixed temporal instant AP3 and TP1 express important principles of the "hole in" relation: without them, it would be hard to put any upper bound on the number of immaterial parasites that can inhabit what - in ordinary thinking - is the region of a single hole. (Some potential counterexamples to TP1 are discussed in [3], the emphasis being on the possibility that two distinct holes may at times be perfectly co-located. However it is not clear whether exact co-location should imply identity of hosts. If we do not make that assumption, AP3 will be a safe and simple identity criterion for the purpose of extensional reasoning. On the other hand, note that this result is only achieved thanks to AP2, which allows one to single out any definable part. In the absence of AP2, or in the context of a different mereological set-up, AP3 might have to be suitably weakened to avoid identifying holes which happen to share a non-separable host.)

In addition to the extensionality axiom, we then have axioms concerning specific patterns of part-whole relations. The following is a basic set:

$$
\begin{array}{ll}
\text { AP4 } & \mathrm{H}(x, y) \rightarrow \neg \mathrm{O}(x, y) \\
\text { AP5 } & \mathrm{H}(x, y) \wedge \mathrm{H}(z, w) \wedge \mathrm{O}(x, z) \rightarrow \mathrm{O}(y, w) \\
\text { AP6 } & \mathrm{H}(x, y) \wedge \mathrm{H}(x, z) \rightarrow \exists_{w}(\mathrm{PP}(w, y \times z) \wedge \mathrm{H}(x, w)) \\
\text { AP7 } & \mathrm{H}(x, y) \wedge \mathrm{H}(z, y) \rightarrow \forall_{w}(\mathrm{HP}(w, x+z) \rightarrow \mathrm{H}(w, y)) .
\end{array}
$$

AP4 expresses the above-mentioned idea that a hole in an object is not a part of that object - indeed, it does not even overlap with it. Of course, this does not rule out that a hole may overlap the host of another hole: the sum of the hole in John's pocket and the slice of Swiss cheese on Mary's plate an entity whose existence is admitted by AP2 - is a scattered individual, partly material and partly immaterial, hosting every hole in that piece of cheese. On this basis, AP5 guarantees that overlapping holes must have overlapping hosts, while the remaining two axioms are essentially meant to ensure that $\mathrm{H}$ and $\mathrm{P}$ interact in the appropriate way given AP4-AP5. By AP6, the common part of any two hosts of a hole must itself be a host of the hole. More specifically, this must be true of some proper part of that common part: there is no way one can pick out a smallest host for a given hole. (This reflects an underlying assumption to the effect that space is dense. Minor adjustments would be required to accommodate different views.) By AP7, we also have it that the common host of two (or more) holes is likewise a 
host of any hole-part thereof. This implies inter alia a form of monotonicity of $\mathrm{H}$ with respect to $\mathrm{P}$ : the hole-parts of a hole are hosted by every host of the hole. This is a form of left monotonicity. In [3] there is also a right monotonicity axiom to the effect that any extension of a hole's host is itself a host of that hole, unless it is a hybrid entity overlapping the hole itself (e.g., the extension obtained by summing the host and part of the hole). The proviso is needed to avoid collision with AP4. But actually something stronger is required: as it stands that axiom would imply for instance that if you take a brick with a hole full of plaster, the given hole is also a hole in the sum brick+plaster - which is false. The correct formulation should strengthen the relevant proviso to the general case of spatial (as opposed to mereological) overlap between the hole and the host's extension. This form of overlap can be treated as a topological relation, and the axiom is therefore postponed to the next section. (See AC7 below.)

Several other important facts follow immediately from AP4-AP7. For example, it follows that a host cannot be part of a hole (a strengthening of AH1), or that mereological atoms (i.e., things that have no proper parts) are bound to be holeless. Here is a sample list of such basic theorems:

$\begin{array}{llr}\text { TP2 } & \mathrm{H}(x, y) \rightarrow-\mathrm{P}(x, y) & \text { (by AP4) } \\ \text { TP3 } & \mathrm{H}(x, y) \wedge \mathrm{H}(x, z) \rightarrow \mathrm{O}(y, z) & \text { (by AP5) } \\ \text { TP4 } & \mathrm{H}(x, y) \rightarrow \exists_{z}(\mathrm{PP}(z, y) \wedge \mathrm{H}(x, z)) & \text { (by AP6) } \\ \text { TP5 } & \mathrm{H}(x, y) \wedge \mathrm{H}(x, z) \rightarrow \neg \mathrm{H}(x, y-z) & \text { (by AP6) } \\ \text { TP6 } & \mathrm{H}(x, y) \wedge \mathrm{P}(y, z) \rightarrow \neg \mathrm{H}(x, z-y) & \text { (by AP6) } \\ \text { TP7 } & \mathrm{H}(x, y) \wedge \mathrm{HP}(z, x) \rightarrow \mathrm{H}(z, y) & \text { (by AP7) } \\ \text { TP8 } & \neg \exists_{z} \mathrm{PP}(z, y) \rightarrow \neg \exists_{x} \mathrm{H}(x, y) . & \text { (by AP6) }\end{array}$

Further stipulative principles can of course be added to strengthen the theory. For instance, in [3] we also have an axiom to the effect that no hole is atomic (though a hole's parts need not necessarily be hole-parts):

AP8 $\quad \mathrm{H}(x, y) \rightarrow \exists_{z} \mathrm{PP}(z, x)$.

This rules out the possibility of treating as a holed object the result, say, of removing a point from a sphere (in agreement with standard topological practice). Holes are spacious, after all. Here, however, I shall confine myself to the core theory defined by AP3-AP7, leaving out any further stipulative principles such as AP8.

2.3. Topology. I see topology as providing a natural next step after mereology in the development of a comprehensive part-whole theory [41, 43]. 
Parthood is a relational concept, wholeness a global property. And in spite of a natural tendency to think of mereology as a theory of parts and wholes, the latter notion cannot be explained in terms of the former. For every whole there is a set of (possibly potential) parts; for every set of parts (i.e., arbitrary objects) there is by AP2 a complete whole, viz. its mereological sum. But there is no way, mereologically, to draw a distinction between "good" and "bad" wholes; there is no way one can distinguish between a one-piece entity, such as a stone or a rope, and a scattered entity made up of various disconnected parts, such as a broken cup or an archipelago (or a soccer tournament, in the temporal realm) by reasoning exclusively in terms of parthood. Thus, the notion of connectedness runs afoul of plain mereology, and a theory of parts and wholes really needs to incorporate a topological machinery of some sort. This becomes apparent especially in connection with qualitative reasoning about space and time: here mereology proves useful to account for certain basic relationships among things or events; but one needs topology to account, say, for the fact that two objects or events can be continuous with each other, or for the relation of something being inside, abutting, or surrounding something else. (My present concern will be with the mereotopology of spatial structures; I have explored eventive and other temporal structures in joint work with Fabio Pianesi [25, 26, 27].)

There are many ways of combining mereology and topology, and the patterns of interaction between these two domains are still a rather underdeveloped field of research. A most neutral way is simply that of adding a topological component to a mereological basis, using for this purpose a new primitive or set of primitives. This is the strategy favored here. Following in the footsteps of [45], the topological primitive will be a binary relation of topological connection (we could as well use the notion of boundary, or that of interior parthood: see [26, 36, 38, 42] for some such alternatives). This relation will be denoted by ' $\mathrm{C}(x, y)$ ' and will be read " $x$ is connected with $y$ ". However, our domain does not merely consist of spatial or spatiotemporal regions but also (rather) of the sort of entities that may inhabit such regions - ordinary entities such as stones, chunks of cheese, holes. Accordingly, connection will be taken to express co-location at (rather than sharing of ) some point in space-time. More precisely, on the intended interpretation something $x$ is connected with something $y$ if and only if either $x$ and the closure of $y$ or $y$ and the closure of $x$ are co-located at some point (where the closure is, as usual, the thing together with its boundary; see [4, 24] for a discussion of the interplay between mereotopology and locative structures). This extension in the meaning of ' $C$ ' is important, as it obviously affects the entire topological machinery to be considered. 
Coming now to the specific format of this topological component, its exact formulation is once again a flexible matter, and various options are in principle available. I shall therefore only spell out the necessary axioms. These include the following two, which ensure that $C$ be reflexive and symmetric (via AP1):

$$
\begin{array}{ll}
\mathrm{AC} 1 & \mathrm{C}(x, x) \\
\mathrm{AC} 2 & \mathrm{P}(x, y) \rightarrow \forall_{z}(\mathrm{C}(x, z) \rightarrow \mathrm{C}(z, y)) .
\end{array}
$$

Many systems developed under the impact of $[7,8]$ also assume the converse of $\mathrm{AC} 2$ (or something to that effect), with the consequence of reducing mereology to topology: see e.g. [1, 28, 29, 30, 31, 32, 44]. However, this is too strong on the present interpretation of $\mathrm{C}$ : an object can be totally inside a hole, hence totally connected with it, without actually sharing any parts with it (think again of the holed piece of Gruyère floating inside a hole in the Emmenthaler). This is indeed the reason why we have to interpret ' $\mathrm{C}$ ' as suggested: insofar as holes are immaterial, they can be interpenetrated by other entities; hence we cannot investigate the topology of the entities in our domain of discourse by studying the topology of the regions that they occupy. Holes do not occupy the region at which they are located.

Given $\mathrm{AC} 1-\mathrm{AC} 2$, we can then define various auxiliary notions such as self-connectedness (SC), spatial enclosure (E), spatial overlapping, or intersection (I), external connection, or abutting $(A)$, superposition (S), interior parthood (IP), tangential parthood (TP), interior enclosure (IE), or tangential enclosure (TE):

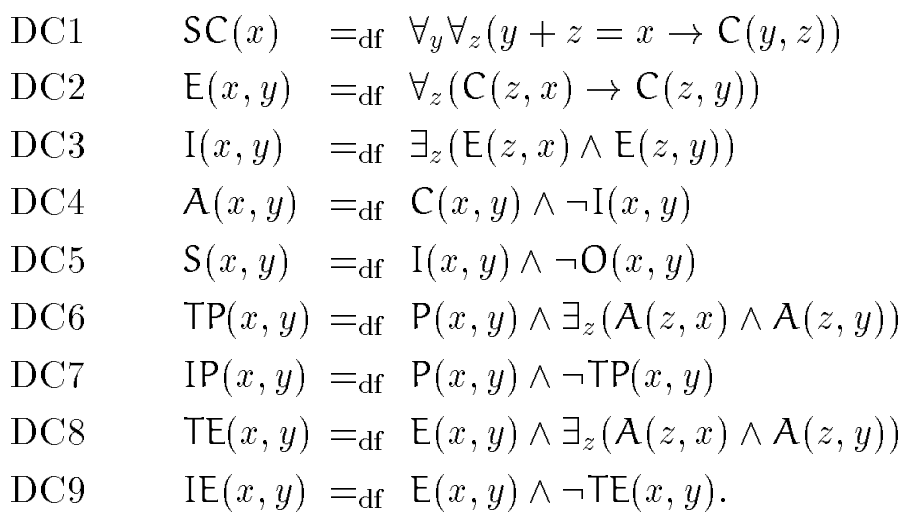

Note that some of these relations are made available precisely by the possibility that topologically connected entities bear no mereological relationship to one another, leaving room for a much richer taxonomy than usually recognized: overlapping is included in, but does not coincide with, inter- 
section, which in turn is properly included in the relation of connection. The basic picture is given in Figure 1 (see [43] for a fuller account). It also bears emphasis that these notions induce a mereotopology on the locative structure corresponding to the entities in our ontology, via the equation $\mathrm{E}(x, y) \Leftrightarrow \mathrm{P}(\mathrm{r}(x), \mathrm{r}(y))$, where $\mathrm{r}(x)$ is the region at which $x$ is exactly located. For more on this I refer to [4].
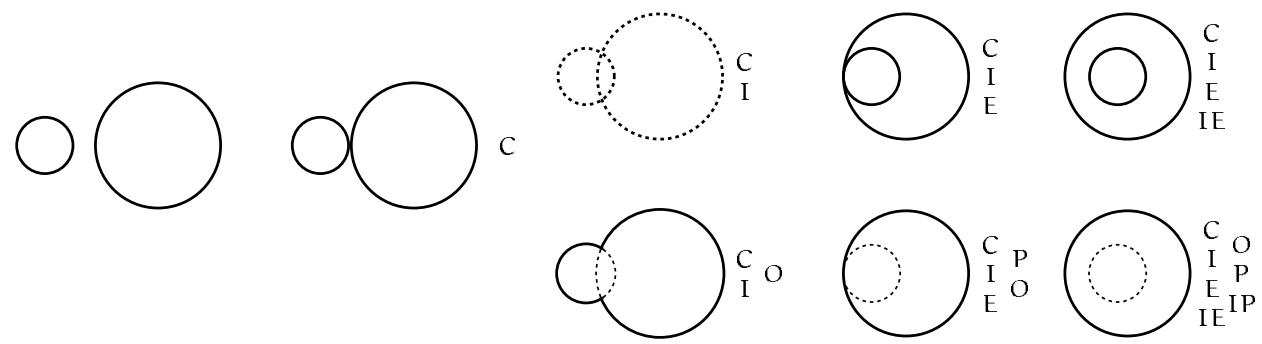

Figure 1. Some basic mereotopological relations (from [26], p. 97); dashed lines indicate actual sharing of parts, as opposed to mere spatiotemporal co-localization.

Moreover, given AP2 the following mereologized versions of the usual notions of interior (i), exterior (e), closure (c), and boundary (b) are legitimately defined:

$\begin{array}{ll}\text { DC10 } & \mathrm{i}(x)={ }_{\mathrm{df}} \quad \sigma y \operatorname{IP}(y, x) \\ \text { DC11 } & \mathrm{e}(x)={ }_{\mathrm{df}} \quad \mathrm{i}(\sim x) \\ \mathrm{DC} 12 & \mathrm{c}(x)={ }_{\mathrm{df}} \sim \mathrm{e}(x) \\ \mathrm{DC} 13 & \mathrm{~b}(x)=_{\mathrm{df}} \sim(\mathrm{i}(x)+\mathrm{e}(x)) .\end{array}$

(Like the operators in DP4-DP9, these operators may be partially defined as we do not assume the existence of a null individual.) Hence we can distinguish between open and closed entities as usual:

$\mathrm{DC} 14 \quad \mathrm{Op}(x)=_{\mathrm{df}} \quad x=\mathrm{i}(x)$

$\mathrm{DC} 15 \quad \mathrm{Cl}(x)=_{\mathrm{df}} x=\mathrm{c}(x)$.

All of these notions are rather well-behaved and allow one to capture a number of important mereotopological notions and facts without however forcing our ontology. To be sure, other axioms would be needed to get reasonably close to a full topological theory. For instance, we can obtain structures corresponding to ordinary topological spaces by imposing (the analogues of) the usual closure conditions: 
AC3

$$
\begin{aligned}
& \mathrm{Cl}(x) \wedge \mathrm{Cl}(y) \rightarrow \mathrm{Cl}(x+y) \\
& \forall_{x}(\varphi \rightarrow \mathrm{Cl}(x)) \rightarrow(z=\pi x \varphi \rightarrow \mathrm{Cl}(z))
\end{aligned}
$$

$\mathrm{AC} 4$

or, equivalently,

$\mathrm{AC}^{\prime} \quad \mathrm{Op}(x) \wedge \mathrm{Op}(y) \rightarrow(z=x \times y \rightarrow \mathrm{Op}(z))$

$\mathrm{AC}^{\prime} \quad \forall x(\varphi \rightarrow \mathrm{Op}(x)) \rightarrow \mathrm{Op}(\sigma x \varphi)$.

In the following, however, I shall not need the full strength of the theory defined by such additional axioms, so I shall not digress here. (See [43] for some material in this direction.)

Moving now to the specific topological or mereotopological axioms for $\mathrm{H}$, I assume the following:

AC5

$$
\mathrm{H}(x, y) \rightarrow \mathrm{SC}(x)
$$

AC6

$$
\mathrm{H}(x, y) \rightarrow \mathrm{A}(x, y)
$$

$\mathrm{AC7}$

$$
\mathrm{H}(x, y) \wedge \mathrm{P}(y, z) \rightarrow(\mathrm{H}(x, z) \vee \mathrm{I}(x, z))
$$

$\mathrm{AC} 8$

$$
\mathrm{H}(x, y) \wedge \operatorname{PHP}(z, x) \rightarrow \Xi_{w}(\mathrm{P}(w, y) \wedge \mathrm{C}(w, x) \wedge \neg \mathrm{C}(w, z)) .
$$

These guarantee that holes are non-scattered entities (AC5) connected to their material hosts (AC6), and that a hole's proper hole-parts (if any) cannot be connected to the very same parts of the host as the hole itself (AC8). (This excludes, for instance, that a single hole be identified with an infinitely descending "pile" of nested holes.) Moreover, AP4 is now derivable from $\mathrm{AC} 6$. As for $\mathrm{AC} 7$, it expresses the intuition, anticipated in the previous section, that holehood be also right monotonic (conditionally) with respect to parthood: typically a mereological extension a hole's host is itself a host of the hole. The conditional proviso can now be properly expressed by requiring that the extended thing be spatially discrete from the hole: it must not overlap the hole, either mereologically (as with the hybrid sum consisting of the host plus the hole) or spatially (as with the sum consisting of the host plus, say, a filler of the hole). This explains the disjunctive form of the consequent of $\mathrm{AC} 7$, which might otherwise collide with AP4 (in case of mereological overlap) or with AC6 (in case of mere superposition). Perhaps there are circumstances in which $\mathrm{AC} 7$ may still sound too strong, for instance in relation to the problematic nature of holes in artifacts. There is a hole in the handle; the handle is part of the door; is the hole also a hole in the door? It is hard to find non-stipulative answers to these questions. But if cases like this are found disturbing, AC7 will have to be further weakened by imposing suitable restrictions on the part-whole relation linking the initial host $y$ and the relevant extension $z$. 
In [3] we also had an axiom to the effect that every hole has a self-connected host: this reflected the intuition that although every hole has infinitely many decreasing hosts (by AP7), which in turn may grow indefinitely (by $\mathrm{AC}$ ), every hole has a privileged host, namely the maximally self-connected one. Every other host would be either a potential part of this one, or a topologically scattered mereological sum including it as a proper part. However, I am now inclined to regard this as too restrictive, i.e., stipulative. Take a piece of cheese with a round cavity hidden inside it, and suppose there is a small round piece of cheese floating right in the middle of the cavity. There are reasons to regard this as an empty cavity in a scattered host, rather than a bigger cavity in a self-connected host partially filled by the small piece. In any event, not much of what follows depends on this point, so the present formulation does not mark a significant departure from the original theory in this respect.

Another hole-specific principle that could be added to AC5-AC8 is that holes are topologically open entities - they are bounded from the outside. This principle was not considered in [3], but is arguably an important feature of holes: they lack a surface of they own; their boundaries belong to their hosts.

AC9 $\quad \mathrm{H}(x, y) \rightarrow \mathrm{Op}(x)$.

One could view this as a topological manifestation of the basic ontological truth about holes: they are parasitic upon the things hosting them. However, the exact formulation of this axiom is not straightforward. Consider the boundary between the hole and that part of its complement which is not occupied by the host. If the hole is open, then that part of its boundary should be part of the complement too. But then consider the complement of the sum hole+host: this complement must be closed in the proximity of the hole, but open elsewhere; for elsewhere the complement is in contact with the host, and the host is closed. (And it follows from our definition that two closed entities cannot be in contact.) This might well be the case, but it would call for an explanation. Alternatively, we could simply say that there is no boundary between the hole and the complement. That is, there is merely a fiat boundary (in the terminology of [37]), as opposed to a bona fide boundary of the usual sort: the hole is an (open) undetached part of the complement. This distinction is detailed elsewhere [42], but it would take us too far afield to spell it out in the present context.

Let us then focus on the basic theory defined by AC5-AC8. Among the new theorems that can be obtained from these axioms, we have then that two holes don't add up to a hole unless they are connected (though the 
converse need not hold: two holes superimposed on each other don't make a hole), that a hole is connected with every host of its hole-parts, that the hole-parts of a hole are all abutting the hosts of that hole, and so on:

TC1 $\quad \mathrm{H}(x+y, z) \rightarrow \mathrm{C}(x, y)$

$\mathrm{TC} 2 \quad \mathrm{H}(x, y) \wedge \mathrm{H}(z, w) \rightarrow \neg \mathrm{P}(y, z)$

TC3 $\mathrm{H}(x, y) \wedge \mathrm{H}(x, z) \rightarrow \mathrm{H}(x, y+z)$

(by $\mathrm{AH} 1+\mathrm{AP} 5+\mathrm{AC} 7)$

(by $\mathrm{AP} 4+\mathrm{AC} 7)$

$\mathrm{TC} 4 \quad \mathrm{H}(x, y) \wedge \mathrm{H}(x, z) \rightarrow \mathrm{H}(x, y \times z)$

(by $\mathrm{AP} 4+\mathrm{AP} 6+\mathrm{AC} 7$ )

TC5 $\quad \mathrm{H}(x, y) \wedge \operatorname{IE}(z, y) \rightarrow \neg \mathrm{H}(x, z))$

TC6 $\quad \mathrm{H}(x, y) \wedge \operatorname{IE}(z, x) \rightarrow \neg \mathrm{H}(z, y)$.

TC7 $\mathrm{H}(x, y) \wedge \mathrm{H}(z, w) \rightarrow(\mathrm{P}(z, x) \rightarrow \mathrm{A}(z, y))$ (by AP4 + AP8 + AC6)

TC8 $\quad \mathrm{H}(x, y) \wedge \mathrm{H}(z, w) \rightarrow(\mathrm{P}(z, x) \rightarrow \mathrm{A}(x, w))$

Moreover, topology now allows us to express certain fundamental differences between holes of different kind. There are three main kinds: superficial holes (or hollows) which correspond to simple depressions or indentations in the surface of the host, and could in principle be eliminated by elastic deformation; perforating holes (tunnels) which introduce non-eliminable topological discontinuities (determining an increase in the topological genus of the host); and internal holes (cavities) which are completely hidden inside the host, and which therefore mark a splitting in the host's complement. These distinctions can be uniformly expressed in terms of the intuitive notion of an opening, or "free face". Let a free face (FF) of a hole $x$, relative to a given host $y$, be a maximally free boundary (FB) of the hole, i.e., a maximally connected part of the hole's (fiat) boundary that is nowhere connected with the host:

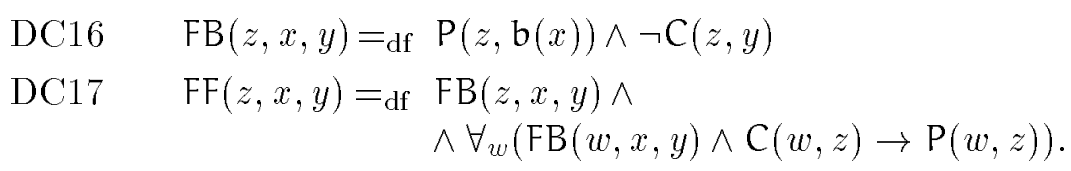

(We shall only have use for these definitions when $x$ and $y$ are as specified.) Then cavities are those holes that have no free faces: the boundary of a cavity is entirely part of the host's boundary, and there is no way you can get out of the cavity without digging through the host. Hollows, on the other hand, do have a free face: you can easily pour water into them. In fact hollows have exactly one free face, and this distinguishes them from full-fledged perforations, which always involve at least two openings - an entrance and an exit, as it were. (If you pour water into them, it can run out from the other side.) Formally, then, the basic distinction between internal holes ( $\mathrm{IH}$ ), 
perforating holes $(\mathrm{PH})$, and superficial holes $(\mathrm{SH})$ may be characterized as follows:

$$
\begin{aligned}
& \mathrm{DC} 18 \quad \mathrm{IH}(x, y)=_{\mathrm{df}} \mathrm{H}(x, y) \wedge \neg \exists_{z} \mathrm{FF}(z, x, y) \\
& \text { DC19 } \mathrm{PH}(x, y)=_{\mathrm{df}} \mathrm{H}(x, y) \wedge \\
& \wedge \exists_{z} \exists_{w}(\mathrm{FF}(z, x, y) \wedge \mathrm{FF}(w, x, y) \wedge \neg \mathrm{C}(z, w)) \\
& \mathrm{DC} 20 \quad \mathrm{SH}(x, y)=_{\mathrm{df}} \mathrm{H}(x, y) \wedge \neg \mathrm{IH}(x, y) \wedge \neg \mathrm{PH}(x, y) \text {. }
\end{aligned}
$$

On this basis several basic facts are easily established. For instance, in addition to various simple properties about cavities (they can only abut things that overlap their hosts, and they must be disconnected from the remaining part of their hosts' complement), we can prove that cavities are maximal holes, in fact maximally connected holes (they cannot be proper parts of other holes and they include every hole with which they are connected), that a hole cannot qualify as a hollow with respect to any part of a host relative to which it already qualifies as a tunnel, or that a hole that qualifies as a tunnel with respect to a hollow's host cannot be part of that hollow (the host would have topologically incompatible properties). Formally:

TC9

$$
\mathrm{IH}(x, y) \rightarrow \neg \mathrm{C}(x, \sim(x+y))
$$

TC10 $\quad \mathrm{IH}(x, y) \wedge A(z, x) \rightarrow \mathrm{I}(z, y)$

TC11 $\quad \mathrm{IH}(x, y) \wedge \mathrm{H}(z, y) \rightarrow \neg \mathrm{PP}(x, z)$

$\mathrm{TC} 12 \quad \mathrm{IH}(x, y) \wedge \mathrm{H}(z, w) \wedge \mathrm{C}(z, x) \rightarrow \mathrm{P}(z, x)$

$\mathrm{TC} 13 \quad \mathrm{PH}(x, y) \wedge \mathrm{SH}(x, z) \rightarrow \neg \mathrm{P}(z, y)$

TC14 $\mathrm{PH}(x, y) \wedge \mathrm{SH}(z, y) \rightarrow \neg \mathrm{P}(x, z)$.

(On the assumption that $x$ is a hole in $y$, some of these conditionals can be strengthened to biconditionals, yielding alternative ways of inducing the basic taxonomy of hole types. For instance, both TC9 and TC10 could serve the purpose of defining internal holes.)
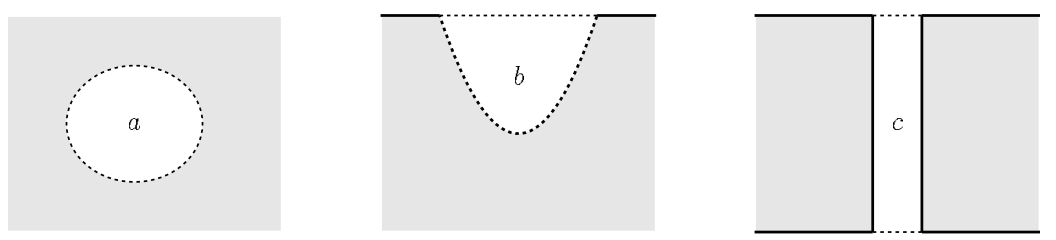

Figure 2. Internal hole, or cavity (a); superficial hole, or hollow (b); perforating hole, or tunnel (c). 
The simplicity of these results is a sign of the expressive power of the hole-based approach. As related work in the area of qualitative topological reasoning makes clear $[13,14]$, the characterization of important topological differences — such as the difference between a sphere and a doughnut seems to run afoul of the basic logic of ' $C$ ' and calls for independent accounts. Explicit reference to holes makes the account straightforward.

2.4. Morphology. The final component of the theory is concerned with concepts and principles that go beyond the confines of part-whole reasoning, showing at the same time the intrinsic limits of mereotopology and the need for more explicit morphological (shape-oriented) analysis. The motivation is simple. The hole taxonomy outlined above reflects certain peculiar topological properties of the host objects which do not, in effect, prevent a uniform treatment: to be a hole is to be a hollow, a tunnel, or a cavity. However, this unity cannot be accounted for by means of mereotopological notions: mereology says nothing at all about it; and topology allows us to distinguish an object perforated by a tunnel (a torus) from an object without (a sphere), but it is of no use to tell a hollowed object from hollowless ones. Topologically, hollows don't count. And the reason is that topology is object-oriented: holes count only insofar as they mark topological discontinuities in their hosts. To account for all the differences, and to appreciate the underlying conceptual unity, we must therefore look directly at the hole, not the object. And the idea behind the morphological component is that we should do so by relying on a fundamental property of holes, arguably their characteristic property: they are fillable. They are spacious and involve concavities, and therefore they can be filled.

Indeed much of our reasoning about holes depends upon our ability to reason about their potential "guests", not only about their actual hosts - a form of complementary reasoning that can be very effective. Of course, there is a sense in which we may properly speak of filling as a relation between a filler (say, a cork) and a material object (the neck of a bottle). In this sense the guest fills the host, not the hole. However, this sense is parasitic on what appears to be a more fundamental notion of filling, whereby some stuff or material body fills (parts of) a hole in another material body. To fill the neck of a bottle is, strictly speaking, to fill the opening of the bottle, which is part of the hole (tunnel) that goes through the neck. It is this relation that we consider here. We indicate it by ' $\mathrm{F}(x, y)$ ', read " $x$ fills $y$ ". In [3] this is taken to express perfect filling, a notion that is intuitively meant to capture the idea that a good filler perfectly heals the concave discontinuity introduced by the hole in its host object. (The healing is defined by the 
minimal surface(s) demarcated by the hole's edge(s), corresponding to the hole's free face(s); I'll come back to this shortly.) We can, however, assume a more general notion of filling, allowing for incomplete fillers as well as for complete but improper (protruding) fillers. Taking ' $F$ ' to express such a general notion, we can then easily define complete, proper, and exact (or perfect) fillers respectively:

$\mathrm{DF} 1 \quad \mathrm{CF}(x, y)=_{\mathrm{df}} \forall_{z}(\mathrm{P}(z, y) \rightarrow \mathrm{F}(x, z))$

$\mathrm{DF} 2 \quad \mathrm{PF}(x, y)=_{\mathrm{df}} \forall_{z}(\mathrm{P}(z, x) \rightarrow \mathrm{F}(z, y))$

DF3 $\quad \mathrm{EF}(x, y)=_{\mathrm{df}} \mathrm{CF}(x, y) \wedge \mathrm{PF}(x, y)$.

Thus, a hole's exact filler can be regarded as the least upper bound (relative to the partial ordering induced by $P$ ) of the hole's proper fillers, or - equivalently — as the greatest lower bound of its complete fillers. The equivalence of this characterization with one taking EF as primitive is thus apparent.
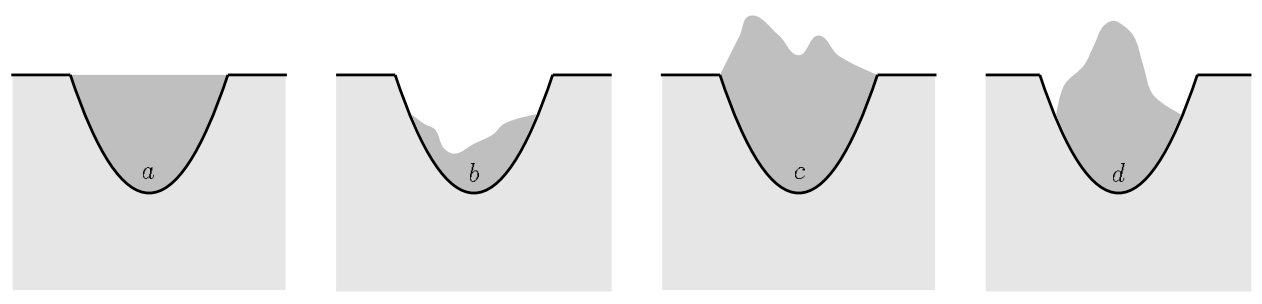

Figure 3. Filling a hole exactly (a); properly, but not completely (b); completely, but not properly $(c)$; generically, i.e., neither completely nor properly $(d)$.

Various specific notions are easily defined using the above. For instance, a filler which, though possibly incomplete, from the outside looks indistinguishable from a complete one (relative to some opening) may be called a lid of the hole. That is, a lid completely covers (hides) some free face of the hole. (A lid may of course be proper or improper, depending on whether it is enclosed in the hole.) Moreover, an interesting intermediate notion is that of a bridge: a lid band connecting two opposite sides of the hole. Likewise, among a hole's fillers we may single out those that act as divisors: they separate the hole into two parts. (There are two kinds: "vertical" and "horizontal" ones, as it were. The former do not separate any part of the hole from its complement; the latter do.) There is no need here to go into such details. But such lines of development are worth mentioning insofar as they are indicative of the conceptual richness implicit in the notion of a filler. 
As general axioms governing the filling relation, and more generally the interplay between morphology and mereotopology, we then assume the following:

$$
\begin{array}{ll}
\text { AF1 } & \mathrm{F}(x, y) \rightarrow \mathrm{S}(x, y) \\
\text { AF2 } & \mathrm{F}(x, y) \wedge \mathrm{E}(y, x) \rightarrow \mathrm{CF}(x, y) \\
\text { AF3 } & \mathrm{F}(x, y) \wedge \mathrm{E}(x, y) \rightarrow \mathrm{PF}(x, y) \\
\text { AF4 } & \exists_{x} \varphi \wedge \forall_{x}(\varphi \rightarrow \mathrm{F}(x, y)) \rightarrow \mathrm{F}(\sigma x \varphi, y) .
\end{array}
$$

AF1 says that filling is a form of superposition: fillers share a location with the filled entities, but there is no sharing of parts. In particular, by AF2 and AF3, the two possible ways in which a hole and its filler may stand in a relation of spatial enclosure correspond to the two main types of filling: holes are enclosed in their complete fillers, and enclose their proper fillers. (Hence, they are exactly co-located with their exact fillers.) This guarantees that proper and complete filling be related in the appropriate way given the underlying mereological structure: a complete filler completely fills every part of a hole, while every part of a proper filler properly fills the hole. Likewise, it follows that every hole is enclosed in its complete fillers and encloses its proper fillers, which implies that a hole's perfect filler can never intersect the hole's own host. Here are the formal renderings of these basic facts, along with the advertised equivalences showing that the choice of ' $F$ ' as a primitive as opposed to ' $E F$ ' is no substantial matter:

$$
\begin{array}{ll}
\text { TF1 } & \mathrm{CF}(x, y) \rightarrow \mathrm{E}(y, x) \\
\text { TF2 } & \mathrm{PF}(x, y) \rightarrow \mathrm{E}(x, y) \\
\text { TF3 } & \mathrm{EF}(x, y) \rightarrow \mathrm{E}(x, y) \wedge \mathrm{E}(y, x) \\
\text { TF4 } & \mathrm{CF}(x, y) \wedge \mathrm{P}(z, y) \rightarrow \mathrm{CF}(x, z) \\
\text { TF5 } & \mathrm{PF}(x, y) \wedge \mathrm{P}(z, x) \rightarrow \mathrm{PF}(z, y) \\
\text { TF6 } & \mathrm{CF}(x, y) \leftrightarrow \exists_{z}(\mathrm{P}(z, x) \wedge \mathrm{EF}(z, y)) \\
\text { TF7 } & \mathrm{PF}(x, y) \leftrightarrow \exists_{z}(\mathrm{P}(z, y) \wedge \mathrm{EF}(x, z)) \\
\text { TF8 } & \mathrm{F}(x, y) \leftrightarrow \exists_{z}(\mathrm{P}(z, x) \wedge \mathrm{PF}(z, y)) \\
\text { TF9 } & \mathrm{F}(x, y) \leftrightarrow \exists_{z}(\mathrm{P}(z, y) \wedge \mathrm{CF}(x, z)) .
\end{array}
$$

As for AF4, this axiom will not play any specific role in the following, but it is added here for the sake of completeness. TF 5 shows that every part of a proper filler is in turn a filler, but of course this form of monotonicity does not hold in the opposite direction: the sum filler+hole, for instance, is not a filler of the hole (by AF1) even if it includes a filler. However, we would 
like to say that in some cases enlarging a filler yields a bigger filler. At the very least, the result of putting together two or more fillers should always yield a filler. This is precisely what AF4 guarantees. Among other things, we have the following closure conditions:

$\begin{array}{ll}\text { TF10 } & \mathrm{PF}(x, y) \wedge \mathrm{PF}(z, y) \rightarrow \mathrm{PF}(x+z, y) \\ \text { TF11 } & \mathrm{CF}(x, y) \wedge \mathrm{CF}(z, y) \rightarrow \mathrm{CF}(x+z, y) .\end{array}$

AF1-AF4 define the basic framework needed to express the idea that holes are fillable entities. There is admittedly much in common between the relations of filling and that of superposition; indeed there are very close and interesting relationships between the fundamental algebra of filling and the structure of spatial location (this is detailed in [4]). Yet the two must be kept separate: superposition is a purely geometric relation which may hold among entities of various kind (among events, for instance, at least according to some theories); filling is a substantial relation expressing the fundamental pattern of interaction between holes and material bodies, and that is what makes it relevant for the purpose of morphological analysis. This is not explicit yet in the general background theory defined by AF1-AF4. But it can be made explicit as soon as we proceed to the specific axioms governing the interplay between the new morphological primitive, $F$, and the fundamental relation of the hole-theory, $\mathrm{H}$ :

$\begin{array}{ll}\text { AF5 } & \mathrm{F}(x, y) \rightarrow \exists_{z} \exists_{w}(\mathrm{H}(z, w) \wedge \mathrm{P}(y, z)) \\ \text { AF6 } & \exists_{z} \exists_{w}(\mathrm{H}(z, w) \wedge \mathrm{P}(x, z)) \rightarrow \neg \mathrm{F}(x, y) .\end{array}$

AF5 expresses the idea that fillability is an exclusive property of holes and parts thereof. Conversely, AF6 says that holes and parts of holes don't fill anything. This is not merely to express the intuition that holes are immaterial. Surely insofar as they are immaterial they cannot fill anything they always leave room for things. But it will be useful to allow for some flexibility in this regard. It will be useful, for instance, to treat the airy plug that always floats inside an "empty" hole as a filler of that hole - a perfect filler, albeit a very feeble one. The reason to exclude holes from the domain of $F$ is, rather, that we want once again to put a clear upper bound on the number of immaterial parasites that can inhabit what - in ordinary thinking - is the region of a single hole. If holes could be fillers, nothing (not even the extensionality axiom AP3) would prevent an infinity of holes to be exactly co-located, each filling the next one in an endless sequence of perfectly superimposed holes. Besides, as we shall see shortly, we must leave 
room for the possibility that a filler be itself holed, and this would collide with AH1 were holes allowed to qualify themselves as bona fide fillers.

Given this, it is obvious that AF1 implies that filling is irreflexive, whereas AF6 implies that it is also asymmetrical and secondarily non-serial: fillables can't fill. Further simple consequences of the axioms are listed below:

TF12 $\neg \mathrm{F}(x, x)$

TF13 $\quad \mathrm{F}(x, y) \rightarrow-\mathrm{F}(y, x)$

TF14 $\quad \mathrm{F}(x, y) \rightarrow-\mathrm{F}(y, z)$

TF15 $\mathrm{H}(x, y) \rightarrow-\mathrm{F}(z, y)$

TF16 $\quad \mathrm{H}(x, y) \rightarrow-\mathrm{F}(y, x)$

$\mathrm{TF} 17 \quad \mathrm{H}(x, y) \wedge \mathrm{EF}(z, x) \rightarrow \mathrm{A}(z, y)$

$\mathrm{TF} 18 \quad \mathrm{H}(x, y) \wedge \mathrm{CF}(z, x) \rightarrow \mathrm{E}(x, z)$

TF19 $\mathrm{H}(x, y) \wedge \mathrm{PF}(z, x) \rightarrow \mathrm{E}(z, x)$ (by $\mathrm{AP} 1+\mathrm{AF} 1$ )

(by $\mathrm{AF} 1+\mathrm{AF} 6$ )

(by $\mathrm{AF} 1+\mathrm{AF} 6$ )

(by AH1 + AP5-6+AF5)

(by AC6 + AF1)

It bears emphasis that nothing prevents the possibility that an exact filler be holed, or that a hole in a complete filler be completely filled by the hole's host. (Think of a wedding ring frozen inside an ice cube: the ring is a holed exact filler of the doughnut-shaped cavity in the cube, and the cube is a complete inexact filler of the hole in the ring.) This is not only intuitively correct. By exploiting this possibility, we can actually improve our basic taxonomy in more than one way. We can distinguish, for instance, between an ordinary, spherical internal cavity and a doughnut-shaped one (a cavity-tunnel in the terminology of [3]): the latter is characterized by the possession of a perfect filler which is doughnut-shaped, hence perforated by a tunnel; the former has a topologically spherical perfect filler. And we can distinguish between these two cases and the case of an internal cavity whose perfect filler involves an internal cavity - a cavity completely filled by (part of) the host of the bigger cavity. (This is a case of a hole with no self-connected host, as discussed above.) We may call these three types of internal holes simple cavities (SIH), doughnut cavities (DIH), and capsule cavities $(\mathrm{CIH})$, respectively:

$\mathrm{DF} 4 \quad \mathrm{DIH}(x, y)=_{\mathrm{df}} \operatorname{IH}(x, y) \wedge \forall_{z}\left(\operatorname{EF}(z, x) \rightarrow \exists_{w} \mathrm{PH}(w, z)\right)$

DF5 $\quad \mathrm{CIH}(x, y)=_{\mathrm{df}} \operatorname{IH}(x, y) \wedge \forall_{z}\left(\operatorname{EF}(z, x) \rightarrow \exists_{w} \operatorname{IH}(w, z)\right)$

DF6 $\quad \operatorname{SIH}(x, y)=_{\mathrm{df}} \operatorname{IH}(x, y) \wedge \neg \operatorname{DIH}(x, y) \wedge \neg \mathrm{CIH}(x, y)$. 

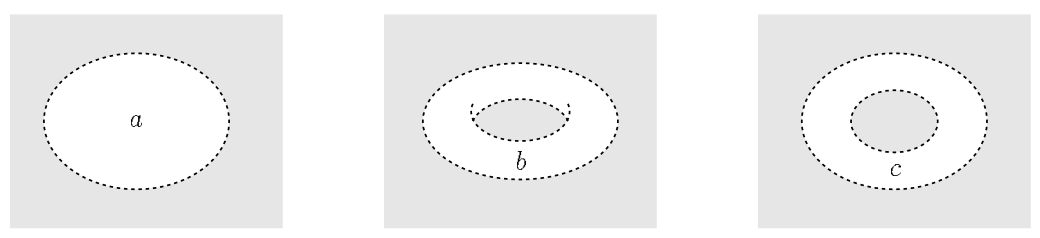

Figure 4. simple cavity $(a)$, a doughnut-cavity $(b)$, and a capsule cavity $(c)$ are distinguished by the topology of their fillers, respectively a ball (holeless), a doughnut (with a tunnel), and a capsule (with an internal cavity).

Similar definitions will allow us to distinguish further subcategories of holes. For instance, if we take $x$ to be a superficial rather than internal hole, then DF4 turns into a characterization of grooves. A groove is a circumcluding hollow (a sort of "open" tunnel): the hole goes all around the host, and its exact filler involves a hole, in fact a perforation (completely but improperly filled by the host). Likewise for the other cases. Indeed the algebra of filling and its various ramifications form an interesting topic of its own. One can investigate, for instance, to what extent the morphological complexity of a hole is mirrored in the topological structure of its "skin", i.e., that part of the host's surface that is externally connected with the hole's exact filler. Further enrichments and refinements of the taxonomy can also be obtained by investigating the many ways holes can be branching or notted together, as suggested in [5]. This shows once again that explicit commitment to holes may have very advantageous consequences: if holes are bona fide entities, one can investigate these aspects by relying explicitly on standard tree and knot theories.

At this point a final remark is in order. I have said that the morphological component of the theory is meant to go beyond mereotopology in the direction of a more shape-oriented account. This should not be taken to suggest that the morphological axioms fix the intended interpretation of the theory so that " $\mathrm{H}(x, y)$ ' really means " $x$ is a hole in (or through) $y$ ". They do not, in fact: the bizarre patterns in Figure $5(-)$ are perfectly compatible with everything said so far. Rather, the axioms are to be taken as specifications of certain basic elucidations of (or constraints on) the notion of a hole, which was and remains the fundamental primitive of the theory. We assume holes to be intuitively understood (recognized, counted, etc.) and we introduce axioms to make explicit certain features that we regard as constitutive of the hole concept. This is the general idea behind the strategy followed here; 
and it is in this sense that the intended model of the theory will not contain holes corresponding to the bizarre shapes depicted in Figure 5.
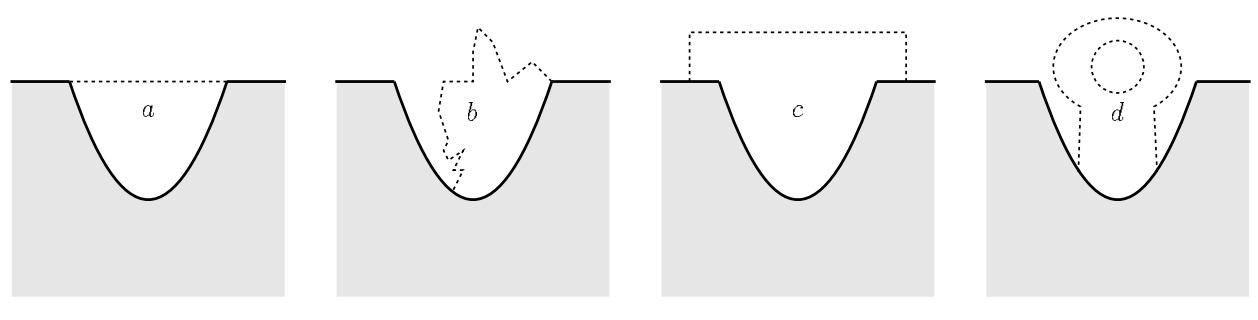

Figure 5. Only $a$ is a hole; $b, c, d$, and the like violate the intuitive condition that a hole's perfect fillers reconstruct the ideal surface of the host object.

Could we rule out such undesired interpretations explicitly? To some extent an answer in the affirmative is possible, provided the language is suitably enriched; but I have no sense of how one can aim at a complete theory. By way of illustration, suppose we add a new primitive operator ' $h(x)$ ' associating each entity $x$ with its "convex hull" - intuitively, the region that would be enclosed if $x$ were wrapped in a taught rubber membrane (Figure 6 ). This operator could be axiomatized along the lines of $[10,31$, 32]:

Ah1 TE $(x, h(x))$

Ah2 $h(h(x))=h(x)$

Ah3 $\quad \mathrm{E}(x, y) \rightarrow \mathrm{P}(\mathrm{h}(x), \mathrm{h}(y))$

Ah4 P $(h(x)+h(y), h(x+y))$

Ah5

$$
\mathrm{P}(\mathrm{h}(x \times y), \mathrm{h}(x) \times \mathrm{h}(y)) .
$$

(These axioms are only indicative and are not meant to form a complete system. Actually the definition of a complete system is - I believe - still an open question; see [9] for a recent assessment.) On this basis we could then add explicit principles aimed at capturing the intuition that holes go hand in hand with concavities. In particular, we could express a basic feature of this intuition by requiring that a hole be always enclosed in the convex hull of its host:

AF7

$$
\mathrm{H}(x, y) \rightarrow \mathrm{E}(x, \mathrm{~h}(y)) .
$$

The undesired patterns in figure 5 will then be ruled out, and only the leftmost pattern will qualify as a case of an object with a hole (a hollow, in effect). 


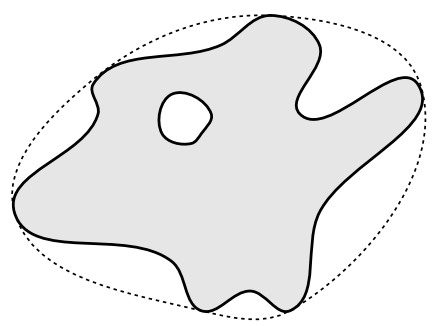

Figure 6. blob with its convex hull. (Here the blob is self-connected, but the notion of a convex hull applies as well to entities that consist of two or more disconnected pieces.)

This way of attacking the issue should not be overestimated. Perhaps it is worth pointing out that AF7, together with AC6, imply the stronger condition that a hole be always enclosed in that part of the convex hull that does not intersect the host, i.e, the difference between the convex hull and the region occupied by the host:

TF20

$$
\mathrm{H}(x, y) \rightarrow \mathrm{E}(x, \mathrm{~h}(y)) \wedge \neg \mathrm{I}(x, y) .
$$

However, this conditional does not hold in the other direction, hence we cannot exploit the convex hull operator to define holes. Obviously this is because not every part of a hole qualifies as a hole, though it is enclosed wherever the hole is. But we cannot rely on the converse of TF20 even if we considered exclusively those parts of $h(y)-\sigma z \mathrm{E}(z, y)$ that are maximally connected. There are two distinct reasons. For one thing, the exact fillers of a hole satisfy exactly the same conditions as the hole itself as far as their location is concerned. An exact filler is enclosed in the very same part of the convex hull as the hole it fills; yet fillers are material bodies, holes immaterial. Secondly, and more importantly, even if we focused exclusively on immaterial bodies, the converse of TF20 would be too strong: a sphere with a horn does not have any hole, yet the result of removing it from its convex hull does leave room for an immaterial body. The best we could say is something like this:

$$
\text { AF8 } \mathrm{E}(x, \mathrm{~h}(y)) \wedge \neg \mathrm{I}(x, y) \wedge \exists_{z} \mathrm{~F}(z, x) \rightarrow \exists_{z}(\mathrm{P}(x, z) \wedge \mathrm{H}(z, y)) .
$$

That is, the fillable entities that are enclosed in the relevant remainder of the convex hull are parts of holes - whence a suitable characterization of hole parts follows using AF6. But of course, this would not serve the purpose of explaining away holes in terms of convex hulls: for the notion of filling is conceptually dependent on that of hole. That is what AF 6 amounts to in the 
first place. (This is clear from the two pairs of patterns in Figure 7. In each case the two patterns satisfy the same conditions; yet one corresponds to a hole, the other does not.) In short, holehood must be assumed as a primitive even if its general geometric earmarks may be fully spelled out. Some holes are just too peculiar, and run afoul of any purely geometric scheme.
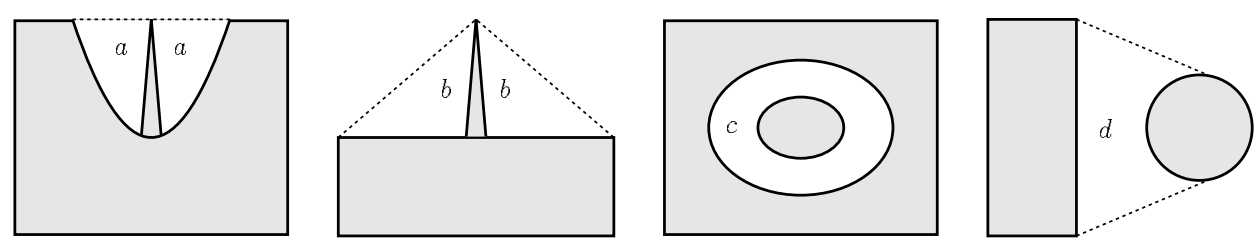

Figure 7. Holes are hard to define in terms of convex hulls: $(a)$ a hole with a spike vs. (b) a spike with no hole; $(c)$ a hole in a scattered host vs. $(d)$ some empty space between two scattered parts.

\section{Examples and Developments: the Geometry of Containment}

The theory sketched above illustrates the level of formalization that can be achieved, as well as the way different domains come to interact, when we set ourselves to spell out a common-sense theory of such ordinary and yet philosophically neglected entities as holes. The result is not only a theory about holes, though. It is also a hole-based theory about space, or more precisely about spatial relations. And the interplay among these different domains involved in the theory (ontology, mereology, topology, and morphology) as well as between these and other domains (such as kinematics or causality) is a fertile line of research not only from a formal ontological perspective, but also for the purpose of spatial reasoning and representation. In the remainder of the paper I would like to illustrate this fact in connection with some indicative examples. In particular, I shall focus on some issues pertaining to the modelling of spatial inclusion. My purpose is mostly taxonomic: I wish to show that explicit commitment to holes can be quite helpful in singling out and keeping track of some relevant distinctions (without pretense of completeness). But I also hope to show that a full-blown theory will enjoy some derivative advantages with regard to both conceptual adequacy and applicative range.

3.1. Insides. A preliminary example coming from natural language processing is provided by the analysis of prepositions of spatial containment 
such as 'in' (or 'inside'). Consider first how our machinery permits a clear account of the idiosyncrasies involved in such arguments as the following:

There is a hole in the brick.

Ex1

The brick is in the wall.

$\checkmark \quad$ There is a hole in the wall.

There is a hole in the cheese.

Ex2

The cheese is in the bucket.

$X$ There is a hole in the bucket.

The different degree of acceptability of these inferences depends on the relationship between the different occurrences of 'in' in the relevant premisses and conclusion. The first argument is non-problematic because it simply reflects the monotonicity of 'in' as a relation of ontological dependence (first premiss) relative to 'in' as a relation of mereological parthood (second premiss) - a principle that here was actually assumed as an axiom (AC7) of the topological segment. (Note how difficult it would be to express this principle in a hole-free language, where the holehood relation is replaced by a holedness predicate.) By contrast, the illegitimacy of the second inference can be explained in terms of failure of the transitivity of 'in' as we move from the relation of ontological dependence (hole in the cheese) to that of spatial containment (cheese in the bucket). In other words, the basic relation $\mathrm{H}$ is monotonic with respect to actual parthood, but not to the (homonymous) relation of containment. Note of course that transitivity is restored if we take both premisses and conclusion to express a relation of containment: in that case it follows unproblematically that the hole is contained in the bucket, though presumably this is not what is ordinarily meant by the sentence in question.

Now, a satisfactory account of 'in' as a relation of spatial containment is by itself an intricate issue. Some authors have suggested an explanation in terms of convex locative inclusion $[15,18,46]$ or mereological inclusion in the convex hull of the containing object $[10,28,30,31,32]$ : something $x$ is in something $y$ just in case (the region occupied by) $x$ is part of the convex hull of (the region occupied by) $y$. Thus, for instance, with reference to the leftmost pattern of Figure 8, the fly is in the glass but the ant is not. As already indicated in [17], however, this approach fails to appreciate the essential role of containing parts as opposed to other non-convex parts. Think of a fly near the stem of a wine glass: it may well fall within the convex hull of the glass, but that does not make it a fly in the glass (Figure 8 ). In other words, reference to the convex hull does not succeed in singling out the regions that are relevant for the purpose of spatial inclusion. Focusing 
exclusively on the convex hull of the object's containing parts (as considered in [39]) is also inadequate. For, apart from the seeming circularity, the outer boundaries of such parts may themselves involve concavities (Figure $8 c)$. This is pointed out in $[1,44]$, where a multi-level account is proposed eventually emphasizing the functional dimension of containment. ([39] also defends a functional approach, further developed in [40].)
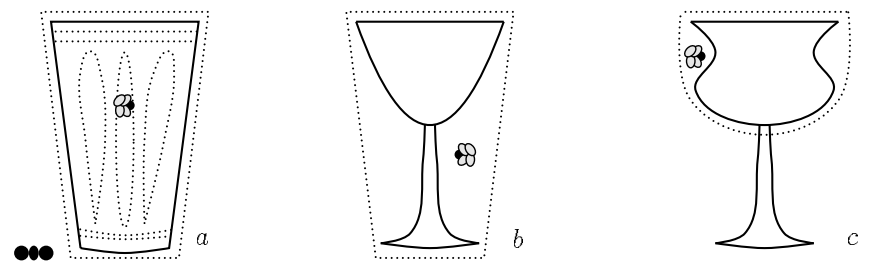

Figure 8. Inclusion in the convex hull is not a criterion for being in the glass ([44], p. 207).

Within the framework outlined above, where holes are accepted as bona fide individuals, the difficulty can be approached perspicuously by relying on the analogy between filling and being in. Roughly, to be contained in an object is to be in a hole of that object; and something is in a hole (wholly or partially) when it can be said to fill the hole (properly or not):

DI1

$$
\operatorname{IN}(x, y) \quad=_{\mathrm{df}} \exists_{z}(\mathrm{H}(z, y) \wedge \mathrm{F}(x, z))
$$

DI2

$$
\mathrm{WIN}(x, y)=_{\mathrm{df}} \forall_{z}(\mathrm{P}(z, x) \rightarrow \mathrm{IN}(z, y))
$$

DI3

$$
\operatorname{PIN}(x, y)=_{\mathrm{df}} \operatorname{IN}(x, y) \wedge \neg \mathrm{WIN}(x, y) \text {. }
$$

Thus, in the patterns corresponding to and $c$ in Figure 8, the fly is not in the glass because it does not fill any hole in the glass. It is located within part of the convex hull of the glass that is not occupied by the glass; but, as we saw, not every such region corresponds to a hole. And only those that do should be taken into account in relation to the question of spatial containment.

In making this suggestion, I take it of course that the containing part of a glass determines a true hole - a hollow, in effect - though there are other senses in which a glass can be said to be holed. (It may have a small perforation in the stem, for instance - I shall come back this shortly.) At the same time, what exactly counts as a hole or a containing part is not at issue here: the account is effective precisely insofar as the existence of independent criteria for holehood is presupposed - e.g., insofar as the space around the stem of a glass is not taken to be a hole. Surely one can imagine to fill up that 
space with plasticine; but we have seen that reasoning exclusively in terms of filling is deceptive: the notion of filling depends essentially on that of a hole. We have a criterion for something to be a filler of a hole, but holes exist and are what they are prior to their fillers. (If we took the space around the stem as a hole, then the fly would be in the glass, on the present account.)

It also bears emphasis that this suggestion is not meant to imply that every case of containment is reducible to hole filling, flexible as the relevant notion of a hole may be. If the glass has a handle, the hole (tunnel) defined by the handle should not count for the purpose of deciding whether the fly is in the glass. Perhaps the stem or the containing part itself is perforated: then, again, a fly in such a hole (perforation) would not be in the glass. Or again, to use a related example from [40], the bulb is in the socket, but the bottle is not in the cap - or so one could argue. (In other cases, filling a hole is not even a necessary condition for containment: think of a bird in the tree [17].) It is apparent that these counterexamples show the limits of the approach insofar as the approach is purely geometric (topological or morphological): a full account calls for a decisive step into other territories. Most likely these include at least some pragmatics, or functional and causal factors at large, as suggested in [1] and [40]. However, the point remains that explicit reference to holes can mark an improvement as far as the geometric part of the story goes. It is true that geometry is not the whole story; but this truth should simply be taken to imply that something else (as I said, a pragmatic or functional explanation) will eventually have to be taken into account. In other words, only some holes count for the purpose of reasoning about containment. But which holes do so count is not a question for the geometric analysis of the problem. (Formally, this means that the bound variable $z$ in DI1 will have to be further restricted, so that only holes of a certain kind and bearing certain relations to the contained object should be taken as admissible values. In the following, however, I shall presume such restrictions to be either forgiveable or tacitly understood.)
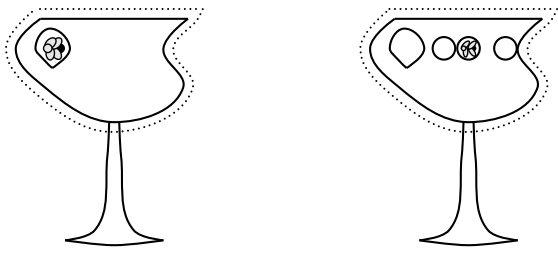

Figure 9. Further difficulties with the relation of containment: in both cases the fly is in a hole hosted by the glass, but not in the glass itself. 
3.2. Containing hulls. On this basis - and within these limits - the picture can be refined in various ways. To begin with, we can relax the requirement that $x$ must fill a hole in $y$ in order for $x$ to be in $y$ : we might want to say that a hole is inside the glass (just like the piece of cheese that hosts it), though we know that holes don't fill anything by AF6. To this end, we may simply extend DI1-DI3 by relying on the relation of intersection (spatial overlapping) rather than filling:

$$
\begin{array}{ll}
\text { DI1 }^{\prime} & \mathrm{IN}^{\prime}(x, y)={ }_{\mathrm{df}} \exists_{z}(\mathrm{H}(z, y) \wedge \mathrm{I}(x, z)) \\
\text { DI2 }^{\prime} & \text { WIN }^{\prime}(x, y)={ }_{\mathrm{df}} \forall_{z}\left(\mathrm{P}(z, x) \rightarrow \mathrm{IN}^{\prime}(z, y)\right) \\
\text { DI3 }^{\prime} & \operatorname{PIN}^{\prime}(x, y)={ }_{\mathrm{df}} \quad \operatorname{IN}^{\prime}(x, y) \wedge \neg \mathrm{WIN}^{\prime}(x, y) .
\end{array}
$$

Of course, this implies that every hole is contained in its host (though immaterially, so to say):

TI1 $\quad \mathrm{H}(x, y) \rightarrow \mathrm{IN}^{\prime}(x, y) \wedge \neg \mathrm{IN}(x, y)$.

Alternatively, we could achieve the same characterization by revisiting the original convex-hull operator (acting on a domain of regions) in terms of a "containing-hull" operator acting directly on objects. In our framework this can be introduced as a function $k$ yielding the fusion of any given object with its own holes:

DI4

$$
\mathrm{k}(x)=_{\mathrm{df}} \sigma z(\mathrm{P}(z, x) \vee \mathrm{H}(z, x)) .
$$

The following equivalences show that the basic notions defined in DI1'-DI3' could then be characterized in terms of this operator:

$$
\begin{array}{ll}
\text { TI2 } & \operatorname{IN}^{\prime}(x, y) \leftrightarrow \mathrm{I}(x, \mathrm{k}(y)-y) \\
\text { TI3 } & \operatorname{WIN}^{\prime}(x, y) \leftrightarrow \mathrm{E}(x, \mathrm{k}(y)-y) \\
\text { TI4 } & \operatorname{PIN}^{\prime}(x, y) \leftrightarrow \mathrm{IN}^{\prime}(x, y) \wedge \neg \operatorname{WIN}^{\prime}(x, y) .
\end{array}
$$

As is might be expected, these equivalences turn out to be analogous to those that can be obtained from the convex-hull-based approach upon replacing convex hulls by containing hulls (see [10] for a comparison). On the other hand, it follows from AF7 that $k(y)$ is always enclosed in $h(y)$ (indeed tangentially enclosed, by Ah1), though the converse may fail:

$$
\operatorname{TE}(k(x), h(y)) .
$$

Thus, only some of the relations defined in terms of $h(y)$ hold upon replacement of ' $h$ ' by ' $k$ '. For instance, $y$ might be a holeless non-convex object 
(such as the block with protruding spike of Figure 7 ), in which case $k(y)$ would be $(=y$ and) properly enclosed in $h(y)$.

Several additional notions could be introduced at this point. In particular, we can immediately make the distinction between generic inside and "just inside"

DI5

$$
\begin{array}{lll}
\text { DI5 } & \text { JIN }(x, y)==_{\mathrm{df}} & \text { WIN }(x, y) \wedge A(\sim \mathrm{k}(y)) \\
\text { DI6 } & \operatorname{JIN}^{\prime}(x, y)=_{\mathrm{df}} & \operatorname{WIN}^{\prime}(x, y) \wedge A(\sim \mathrm{k}(y)) .
\end{array}
$$

and we can make the picture more complete by defining also the relations of being generically, wholly, or just "outside" a given object.

$$
\begin{aligned}
& \text { DI7 OUT }(x, y) \quad=_{\mathrm{df}} \neg \mathrm{I}(x, \mathrm{k}(y)) \\
& \text { DI8 JOUT }(x, y)={ }_{\mathrm{df}} \mathrm{A}(x, \mathrm{k}(y)) \\
& \text { DI9 } \quad \text { WOUT }(x, y)=_{\mathrm{df}} \operatorname{OUT}(x, y) \wedge \neg \operatorname{JOUT}(x, y) \text {. }
\end{aligned}
$$

Thus, an object is properly inside another if and only if no part of it is outside, and it is outside if and only if no part is inside:

TI6

TI7

$$
\begin{aligned}
& \operatorname{PIN}^{\prime}(x, y) \leftrightarrow \forall_{z}(\mathrm{P}(z, x) \rightarrow \neg \operatorname{OUT}(z, y)) \\
& \operatorname{OUT}(x, y) \leftrightarrow \forall_{z}\left(\mathrm{P}(z, x) \rightarrow \neg \operatorname{PIN}^{\prime}(z, y)\right) .
\end{aligned}
$$

The natural transition from the two extreme positions, corresponding to the relations WOUT and WIN' (or WIN, for that matter), is illustrated in Figure 10.
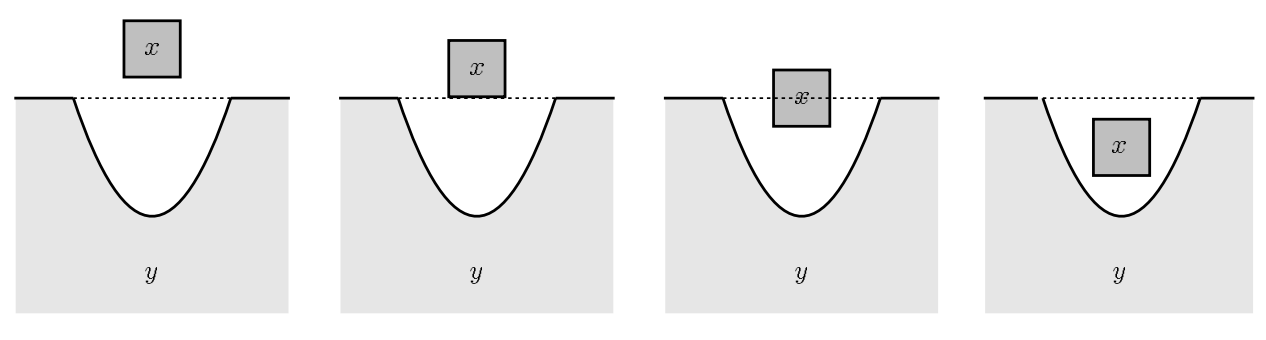

Figure 10. Natural transition of an object $x$ from wholly outside (left) to wholly inside (right) a hollowed object $y$.

Note that none of the relations thus defined is fully transitive. They are not asymmetric either, except for WIN and JIN, and of course they are not reflexive. On the other hand, IN ( $\left.\mathrm{IN}^{\prime}\right)$, WIN (WIN'), OUT, and WOUT satisfy certain basic forms of dissectivity or monotonicity that have 
no analogue for PIN (PIN') or JOUT: for instance, it is easily verified that the parts of whatever is wholly inside or (wholly) outside an object $y$ are also so related to $y$, although what is (partially) in $y$ may obviously have parts that lie outside $y$, and what is just outside $y$ may have parts wholly outside it:

$$
\begin{array}{ll}
\text { TI8 } & \neg \mathrm{IN}(x, x) \\
\text { TI9 } & \neg \text { OUT }(x, x) \\
\text { TI10 } & \text { WIN }(x, y) \rightarrow-\operatorname{WIN}(y, x) \\
\text { TI11 } & \operatorname{JIN}(x, y) \rightarrow \neg \operatorname{JIN}(y, x) \\
\text { TI12 } & \operatorname{JIN}(x, y) \wedge \operatorname{JOUT}(z, y) \rightarrow \neg \mathrm{I}(x, z) \\
\text { TI13 } & \operatorname{IN}(x, y) \wedge \mathrm{P}(z, x) \rightarrow \operatorname{IN}(z, y) \\
\text { TI14 } & \operatorname{IN}^{\prime}(x, y) \wedge \mathrm{P}(z, x) \rightarrow \operatorname{IN}^{\prime}(z, y) \\
\text { TI15 } & \text { WiN }(x, y) \wedge \mathrm{P}(z, x) \rightarrow W i N(z, y) \\
\text { TI16 } & \text { WOUT }(x, y) \wedge \mathrm{P}(z, x) \rightarrow W O U T(z, y) .
\end{array}
$$

It is immediately seen that this characterization can then be further specialized to support richer taxonomies such as the ones introduced in [10, $30,32]$, which are based on the general convex-hull approach mentioned above. The generating schema is the following:

DI10

$$
\Phi-\Psi-R(x, y)=_{\mathrm{df}} \Phi(x, y) \wedge \Psi(y, x) \wedge \neg \mathrm{R}(x, y),
$$

where in general $\Phi$ and $\Psi$ are any of the containment relations defined above and $R$ is any of $O, S$, or $C$. I leave the computation of the number of distinct relations generated by D19 to the reader. Further notions can be obtained by fully exploiting the algebra of spatial location, as indicated in [4].

3.3. Further modes of containment. As I said, it is not claimed that the relations introduced above allow for a full account of the notion of spatial containment, or even for a full solution of the fly-in-the glass problem. Even so, several useful refinements can be introduced already at the geometrical level, including some applications to naive-physical reasoning about containment (in the spirit of [16]). For instance, we have already seen that we can distinguish between an inside that involves true "filling" (as in DI1) from that of a "vacuous", purely geometrical inside:

DI11 VIN $(x, y)=_{\mathrm{df}} \operatorname{IN}^{\prime}(x, y) \wedge \neg \mathrm{IN}(x, y)$.

The latter, but not the former, would apply to the case of the small hole inside a bigger one. 
Likewise, in both cases we can account for the distinction between generic inside and strict topological inside, the latter occurring only in the presence of internal holes. And we may distinguish between partial and whole topological inside depending on whether the guest object is partially or wholly enclosed in the relevant hole, or, more generally, whether or not every part of the guest object is topologically inside the host object. The definition are as follows

$$
\begin{array}{ll}
\text { DI12 } & \text { TIN }(x, y)==_{\mathrm{df}} \exists_{z}(\operatorname{IH}(z, y) \wedge \mathrm{F}(x, z)) \\
\text { DI13 } & \text { WTIN }(x, y)=_{\mathrm{df}} \forall_{z}(\mathrm{P}(z, x) \wedge \operatorname{TIN}(z, y)) \\
\text { DI14 } & \operatorname{PTIN}(x, y)={ }_{\mathrm{df}} \quad \operatorname{TIN}(x, y) \wedge \neg \mathrm{WTIN}(x, y) .
\end{array}
$$

(The corresponding notions for the vacuous case can be obtained by putting ' $I$ ' in place of ' $F$ ' in DI12, though it probably makes little intuitive sense to speak of an immaterial entity such as a hole being forcefully kept inside another hole: if it so happens, it is because its host is kept inside. Similar remarks apply to the notions defined below, which will only be given in terms of filling inside.) Of course this implies that topological inside can never be partial unless the guest is scattered, and that something is wholly topologically inside an object only if it cannot move to the outside without cutting through the object itself (think of a maggot eating its way out of a cavity in a wheel of Swiss cheese):

$$
\operatorname{TIN}(x, y) \wedge \mathrm{SC}(x) \rightarrow W \operatorname{WTN}(x, y)
$$

TI18 $\operatorname{WTIN}(x, y) \rightarrow \forall_{z}(\mathrm{SC}(z) \wedge \mathrm{C}(z, x) \wedge \mathrm{C}(z, \sim \mathrm{k}(y)) \rightarrow \mathrm{I}(z, y))$.

In a similar manner we can account for other cases in which the guest object cannot be let free without cutting. Topological inside define one such case (Figure 11a); another is what may be labelled "constrained inside" ( ): the hole is open and the object inside it is or can be put in contact with the outside, but the presence of a topological discontinuity constraints its freedom to move.

DI15

$$
\begin{aligned}
\operatorname{CIN}(x, y))_{\mathrm{df}} & \exists_{z}\left(\mathrm{H}(z, y) \wedge \mathrm{F}(x, z) \wedge \forall_{u}(\operatorname{EF}(w, z) \rightarrow\right. \\
& \left.\left.\rightarrow \exists_{u}(\operatorname{IH}(u, w) \wedge \operatorname{IE}(u, \mathrm{k}(y)) \wedge \mathrm{E}(u, \mathrm{k}(x)))\right)\right) .
\end{aligned}
$$

From a naive-physical perspective this is perhaps one of the most important patterns of interaction relating to holes: keeping material objects (fillers) in place, or at least hindering their movement. There are of course many other such patterns besides topological constraint. For instance, a plugger is typically a complete, non-exact filler some parts of which are externally 
connected with the host but not with the hole, so that its translational freedom [33] is constrained. Or take the last two cases depicted in Figure 11 $(c, \quad)$. Here the guest object is kept inside the host by virtue of morphological constraints. Unlike $a$ and, the guest could be let free without altering the topology; but unlike the basic pattern in Figure 10, $x$ can be let free only if $y$ or $x$ itself is suitably deformed. Again, we reach here a point where pure mereotopological reasoning shows its limits - shapes become crucial. And shapes are not the end of the story either. A square complete filler will be kept in place by its square hole, but a round filler will rotate in its circular hole. One would need here to investigate the frictional properties of the stuff of both host and guest, and an explicit step into the territory of naive kinematics seems required in order to capture the relevant distinctions. This, I believe, is a promising subject for further exploration.
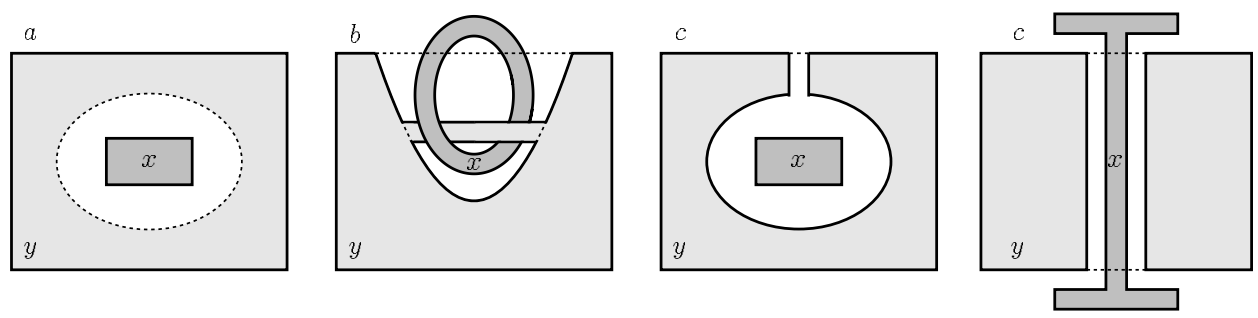

Figure 11. Patterns of constrained inside. In some cases the constraint is purely topological $(a, b)$; other cases depend crucially on the morphology of the contained and containing objects $(c, d)$.

Acknowledgments. This paper stems from joint work with Roberto Casati, with whom I shared virtually every single thought I had on this topic. A very preliminary and much shorter version appeared as "Spatial Reasoning in a Holey World" in Pietro Torasso (ed.), Advances in Artificial Intelligence (Lecture Notes in Artificial Intelligence 728, Berlin/Heidelberg/New York: Springer-Verlag, 1993, pp. 326-336) and is partly reproduced here by kind permission of Springer-Verlag GmbH \& Co. Preliminary versions were also delivered at conferences in Chambery, Paris, Trento, Buffalo, Bloomington, and Bielefeld; I am thankful to participants for stimulating discussion. I also owe much to conversations I enjoyed with Stefano Borgo, Tony Cohn, Antony Galton, Nicola Guarino, Wolfgang Heydrich, Claudio Masolo, Sidney Morgenbesser, Jerzy Perzanowski, Peter Simons, Barry Smith, and Claude Vandeloise. 


\section{References}

[1] Aurnague M., Vieu L., "A Three-Level Approach to the Semantics of Space", [in:] C. Z. Wibbelt (ed.), The Semantics of Prepositions: From Mental Processing to Natural Language Processing, Berlin: Mouton de Gruyter, 1993, pp. 393-439.

[2] Bäckström C., "Logical Modelling of Simplified Geometrical Objects and Mechanical Assembly Processes", [in:] Su-shing Chen (ed.), Advances in Spatial Reasoning, Volume 1, Norwood: Ablex, 1990, pp. 35-61.

[3] Casati R., Varzi A. C., Holes and Other Superficialities, Cambridge, MA, and London: MIT Press (Bradford Books), 1994.

[4] Casati R., Varzi A. C., "The Structure of Spatial Localization", Philosophical Studies, 82 (1996), 205-239.

[5] Casati R., Varzi A. C., "Spatial Entities", [in:] O. Stock (ed.), Spatial and Temporal Reasoning, Dordrecht/Boston/London: Kluwer, forthcoming.

[6] Chisholm R. M., "Boundaries as Dependent Particulars", Grazer Philosophische Studien, 10 (1984), 87-95.

[7] Clarke B. L., "A Calculus of Individuals Based on 'Connection'”, Notre Dame Journal of Formal Logic, 22 (1981), 204-218.

[8] Clarke B. L., "Individuals and Points", Notre Dame Journal of Formal Logic, 26 (1985), 61-75.

[9] Cohn A. G., "Qualitative Shape Representation using Connection and Convex Hulls", [in:] P. Amsili, M. Borillo, and L. Vieu (eds.), Time, Space and Movement: Meaning and Knowledge in the Sensible World. Proceedings of the 5th International Workshop, Toulouse: COREP, Part C, pp. 3-16.

[10] Cohn A. G., Randell D. A., Cui Z., "A Taxonomy of Logically Defined Qualitative Spatial Regions" , International Journal of Human-Computer Studies, 43 (1995), 831-846.

[11] Davis E., "A Framework for Qualitative Reasoning About Solid Objects", [in:] G. Rodriguez (ed.), Proceedings of the Workshop on Space Telerobotics, Pasadena, Ca.: NASA and JPL, 1987, pp. 369-375.

[12] Fine K., "Part-Whole", [in:] B. Smith and D. W. Smith (eds.), The Cambridge Companion to Husserl, New York: Cambridge University Press, 1995 , pp. $463-485$. 
[13] Gotts, N. M., "How Far Can We 'C'? Defining a 'Doughnut' Using Connection Alone", [in:] J. Doyle, E. Sandewall, and P. Torasso (eds.), Principles of Knowledge Representation and Reasoning: Proceedings of the Fourth International Conference, San Mateo, CA: Morgan Kaufmann, 1994, pp. 246-257.

[14] Gotts, N. M., "Defining a 'Doughnut' Made Difficult", [in:] C. Eschenbach, C. Habel, and B. Smith (eds.), Topological Foundations of Cognitive Science. Papers from the Workshop at the First International Summer Institute in Cognitive Science, University of Hamburg, Reports of the Doctoral Program in Cognitive Science, No. 37, 1994, pp. 105-129.

[15] Habel C., "Zwischen-Bericht", [in:] C. Habel, M. Herweg, and K. Rehkämper (eds.), Raumkonzepte in Verstehenprozessen, Tübingen: Niemeyer, 1989, pp. $37-69$.

[16] Hayes P. J., "Naive Physics I: Ontology for Liquids", [in:] J. R. Hobbs and R. C. Moore (eds.), Formal Theories of the Commonsense World, Norwood: Ablex, 1985, pp. 71-107.

[17] Herskovits A., Language and Spatial Cognition. An Interdisciplinary Study of the Prepositions in English, Cambridge: Cambridge University Press, 1986.

[18] Herweg M., "Ansätze zu einer semantischen Beschreibung topologischer Präpositionen", [in:] C. Habel, M. Herweg, and K. Rehkämper (eds.), Raumkonzepte in Verstehenprozessen, Tübingen: Niemeyer, 1989, pp. 99-127.

[19] Hoffman D., Richards W. A., "Parts of Recognition", Cognition, 18 (1985), $65-96$.

[20] Jackson F., Perception. A Representative Theory, Cambridge: Cambridge University Press, 1977.

[21] Lewis D. K., Lewis S. R., "Holes", Australasian Journal of Philosophy, 48 (1970), 206-212.

[22] Leonard H. S., Goodman N., "The Calculus of Individuals and Its Uses", Journal of Symbolic Logic, 5 (1940), 45-55.

[23] Martin R. M., Events, Reference, and Logical Form, Washington: Catholic University of America Press, 1978.

[24] Perzanowski J., "Locative Ontology. Parts I-III", Logic and Logical Philosophy, 1 (1993), 7-94.

[25] Pianesi F., Varzi A. C., "The Mereo-Topology of Event Structures", [in:] P. Dekker and M. Stokhof (eds.), Proceedings of the 9th Amsterdam Colloquium, Amsterdam: ILLC, 1994, pp. 527-546. 
[26] Pianesi F., Varzi A. C., "Events, Topology, and Temporal Relations", The Monist, 79 (1996), 89-116.

[27] Pianesi F., Varzi A. C., "Refining Temporal Reference in Event Structures", Notre Dame Journal of Formal Logic, 37 (1996), 71-83.

[28] Randell D. A., Analysing the Familiar: Reasoning about Space and Time in the Everyday World, University of Warwick: PhD Thesis, 1991.

[29] Randell D. A., Cohn A. G., "Modelling Topological and Metrical Properties in Physical Processes", [in:] R. J. Brachman, H. J. Levesque, and R. Reiter (eds.), Principles of Knowledge Representation and Reasoning. Proceedings of the First International Conference, Los Altos: Morgan Kaufmann, 1989, pp. 357-368.

[30] Randell D. A., Cohn A. G., "Exploiting Lattices in a Theory of Space and Time", Computers and Mathematics with Applications, 23 (1992), 459-476.

[31] Randell D. A., Cui Z., Cohn A. G., "An Interval Logic of Space Based on 'Connection'", [in:] B. Neumann (ed.), Proceedings of the 10th European Conference on Artificial Intelligence, Chichester: John Wiley \& Sons, 1992, pp. 394-398.

[32] Randell D. A., Cui Z., Cohn A. G., "A Spatial Logic Based on Regions and Connections", [in:] B. Nebel, C. Rich, and W. Swartout (eds.), Principles of Knowledge Representation and Reasoning. Proceedings of the Third International Conference, Los Altos: Morgan Kaufmann, 1992, pp. 165-176.

[33] Shoham Y., "Naive Kinematics: Two Aspects of Shape", [in:] J. R. Hobbs (ed.), Commonsense Summer: Final Report, Technical Report \# CSLI-85-35, Stanford: SRI International, AI Center, 1984, pp. 4:1-25.

[34] Simons P. M., Parts. A Study in Ontology, Oxford: Clarendon, 1987.

[35] Simons P. M., "Free Part-Whole Theory", [in:] K. Lambert (ed.), Philosophical Applications of Free Logic, Oxford/New York: Oxford University Press, 1991, pp. 285-306.

[36] Smith B., "Ontology and the Logistic Analysis of Reality", [in:] G. Häfliger and P. M. Simons (eds.), Analytic Phenomenology, Dordrecht - Boston London: Kluwer, forthcoming.

[37] Smith B., "Fiat Objects," [in:] N. Guarino, S. Pribbenow, and L. Vieu (eds.), Parts and Wholes: Conceptual Part-Whole Relations and Formal Mereology. Proceedings of the ECA194 Workshop, Amsterdam: European Coordinating Commettee for Artificial Intelligence, 1994, pp. 15-23.

[38] Tiles J. E., Things That Happen, Aberdeen: Aberdeen University Press, 1981. 
[39] Vandeloise C., L'espace en français: sémantique des prépositions spatiales, Paris: Seuil, 1986 (Eng. trans. by A. R. K. Bosch, Spatial Prepositions. A Case Study from French, Chicago: University of Chicago Press, 1991).

[40] Vandeloise C., "Methodology and analyses of the preposition in", Cognitive Linguistics, 5 (1994), 157-184.

[41] Varzi A. C., "On the Boundary Between Mereology and Topology", [in:] R. Casati, B. Smith, and G. White (eds.), Philosophy and the Cognitive Sciences. Proceedings of the 16th International Wittgenstein Symposium, Vienna: Hölder-Pichler-Tempsky, 1994, pp. 423-442.

[42] Varzi A. C., "Boundaries, Continuity, and Contact", Noûs, forthcoming.

[43] Varzi A. C., "Parts, Wholes, and Part-Whole Relations: The Prospects of Mereotopology", Data \& Knowledge Engineering, forthcoming.

[44] Vieu L., Sémantique des relations spatiales et inférences spatio-temporelles: Une contribution à l'étude des structures formelles de l'espace en Langage Naturel, Université Paul Sabatier de Toulouse: PhD Thesis, 1991.

[45] Whitehead A. N., Process and Reality. An Essay in Cosmology, New York: Macmillan, 1929.

[46] Wunderlich D., "Raum, Zeit, und das Lexicon", [in:] H. Schweizer (ed.), Sprache und Raum, Stuttgart: Metzler, 1985, pp. 66-89.

ACHILle C. VARZI

Department of Philosophy

Columbia University

New York, NY 10027, USA

e-mail: av72@columbia.edu 